\title{
Similarities and differences in uterine gene expression patterns caused by treatment with physiological and non-physiological estrogens
}

\author{
H Watanabe, A Suzuki, M Kobayashi, D B Lubahn', H Handa ${ }^{2}$ and T Iguchi \\ Center for Integrative Bioscience, Okazaki National Research Institutes and Core Research for Evolution Science and Technology (CREST), Japan \\ Science and Technology Corporation, 5-1 Higashiyama, Myodaiji, Okazaki 444-8585, Japan \\ ${ }^{1}$ Department of Molecular Biology, University of Missouri, Columbia, Missouri 65211, USA \\ ${ }^{2}$ Frontier Collaborative Research Center, Tokyo Institute of Technology, 4259 Nagatsuta-cho, Yokohama, Kanagawa 226-8503, Japan
}

(Requests for offprints should be addressed to T Iguchi; email: taisen@nibb.ac.jp)

\begin{abstract}
Administration of physiological and non-physiological estrogens during pregnancy or after birth is known to have adverse effects on the development of the reproductive tract and other organs. Although it is believed that both estrogens have similar effects on gene expression, this view has not been tested systematically. To compare the effects of physiological (estradiol; E2) and non-physiological (diethylstilbestrol; DES) estrogens, we used DNA microarray analysis to examine the uterine gene expression patterns induced by the two estrogens. Although E2 and DES induced many genes to respond in the same way, different groups of genes showed varying levels of maximal activities to each estrogen, resulting in different dose-response patterns. Thus, each estrogen has a distinct effect on uterine gene expression. The genes were classified into clusters according to their dose-responses to the two estrogens. Of the eight clusters, only two correlated well with the uterotropic effect of different doses of E2. One of these clusters contained genes that were upregulated by E2, which included genes encoding several stress proteins and transcription factors. The other cluster contained genes that were downregulated by E2, including genes related to metabolism, transcription and detoxification processes. The expression of these genes in estrogen receptor-deficient mice was not affected by E2 treatment, indicating that these genes are affected by the E2-bound estrogen receptor. Thus, of the many genes that are affected by estrogen, it was suggested that only a small number are directly involved in the uterotropic effects of estrogen treatment.
\end{abstract}

Journal of Molecular Endocrinology (2003) 31, 487-497

\section{Introduction}

Diethylstilbestrol (DES), a non-physiological estrogen, was developed in the late 1930s for clinical use and is thought to mimic the effect of estradiol (E2) (Dodds et al. 1938). Subsequently, it was found that treatment of human fetuses and neonatal mice with DES as well as with physiological estrogens causes reproductive tract abnormalities and vaginal adenosis (Kimura et al. 1967). It is believed that treatment with physiological and non-physiological estrogens results in these reproductive abnormalities because they both act at the wrong time during development. As DES and E2 have a high affinity for the estrogen receptor and both show uterotropic effects, it has been assumed that both estrogens regulate gene expression in the same way. However, this notion has not been extensively tested.

To examine the effect of physiological and non-physiological estrogens on gene expression, we used DNA microarray analysis. DNA microarray technology has been successfully applied to characterize the genome-wide gene expression that results from various stimuli, such as hormone treatment (Feng et al. 2000, Flores-Morales et al. 2002). High-density oligonucleotide arrays (Lockhart et al. 1996) are especially suitable for genome-wide mapping of gene expression because a large number of genes can be analyzed at one time, and the system 
can be readily scaled up to allow the simultaneous monitoring of tens of thousands of genes.

In this study, gene expression changes in the uterus following physiological (E2) and nonphysiological (DES) estrogen treatment were profiled using DNA microarrays. The uterus has long been used as a measure of estrogenicity because the horn size and uterine weight correlate well with the dose of estrogen administered. Clustering analysis of the gene expression pattern revealed similar gene expression profiles for E2 and DES. However, the expression of some genes were more greatly affected by DES than by E2, and vice versa. The expression of these genes were not altered by estrogen treatment of estrogen receptor $(\mathrm{ER}) \alpha$ knockout $(\alpha \mathrm{ERKO})$ mice, which suggests that ER is involved in the estrogenic effects of DES and E2. In addition, by assessing the correlation between the gene expression and the uterotropism caused by various doses of estrogen, we were able to select the genes that appear to be involved in the uterotropic effects of estrogen treatment.

\section{Materials and methods}

\section{Animals}

Animals were housed under a $12 \mathrm{~h}$ light/12 h darkness cycle. $\alpha \mathrm{ERKO}$ mice (Lubahn et al. 1993) and their wild-type counterparts (C57/BL6/J background) were ovariectomized at eight weeks of age. Two weeks later, the ovariectomized mice were injected intraperitoneally with estrogen or sesame oil (Nakarai Tesque, Kyoto, Japan) as a vehicle control and whole uteri $(n=4)$ were collected $6 \mathrm{~h}$ later. Estrogen doses used were 50, 5, 0.5 and $0.05 \mu \mathrm{g} / \mathrm{kg}$ body weight (b.w.) for $17 \beta$-estradiol (E2) (Sigma-Aldrich Japan, Tokyo, Japan), and 5 and $0.5 \mu \mathrm{g} / \mathrm{kg}$ b.w. for DES (Sigma-Aldrich Japan). For the uterotropic assay, the ovariectomized mice were injected intraperitoneally with estrogen or sesame oil every $24 \mathrm{~h}$ for 7 days and whole uteri $(n=5)$ were collected and weighed. All animal experiments were approved by the institutional Animal Care Committee.

\section{Preparation of labeled cRNA and hybridization}

Total uterine RNA was extracted using the TRIZOL reagent (Invitrogen, Tokyo, Japan) and purified using the RNeasy total RNA purification kit (Qiagen, Tokyo, Japan). Total RNA (10 $\mu \mathrm{g}$ ) was converted into double-stranded cDNA using the SuperScript Choice System (Invitrogen) except that the T7-(dT) 24 primer (Amershambiosciences, Tokyo, Japan) was used for first-strand cDNA synthesis. Biotin-labeled cRNA was synthesized using the ENZO BioArray HighYield RNA transcript labeling kit (Amershambiosciences). The cRNA was purified using RNeasy (Qiagen) and partly hydrolyzed in fragmentation buffer $(40 \mathrm{mM}$ Tris (pH 8.1), $100 \mathrm{mM}$ potassium acetate, and $30 \mathrm{mM}$ magnesium acetate) by $94^{\circ} \mathrm{C}$ heat treatment for $35 \mathrm{~min}$. The fragmented cRNA was mixed with hybridization buffer containing $100 \mathrm{mM}$ 2-(N-Morpholino)ethanesulfonic acid, $1 \mathrm{M} \mathrm{NaCl}, 20 \mathrm{mM}$ EDTA and $0.01 \%$ Tween 20 and control oligonucleotides. The quality of the cRNA was assessed by analysis with the Test 3 array (Affymetrix, Amershambiosciences) containing housekeeping genes. All preparations met Affymetrix's recommended criteria for use on their expression arrays.

After checking the quality of the cRNAs, $15 \mu \mathrm{g}$ cRNA were hybridized to the high-density oligonucleotide arrays (Mouse U74 A, Affymetrix, Amershambiosciences) for $16 \mathrm{~h}$ at $45^{\circ} \mathrm{C}$. The arrays were then washed, stained with streptavidinphycoerythrein (Molecular Probes, Funakoshi, Tokyo Japan) and scanned with an argon-ion laser confocal scanner (Amershambiosciences).

\section{Microarray data analysis}

Scanned data were analyzed with GeneChip software (Affymetrix, Amershambiosciences). The details of the methods used to analyze the data have been described (Lockhart et al. 1996). Briefly, each gene is represented by 20 perfectly matched (PM) and one-base-mismatched (MM) 25-base oligonucleotides. As the MM probes are used to detect background levels and cross-hybridization signals, the relative level of gene expression is represented by the differences between the levels of fluorescent intensity of the PMs and the MMs, which are averaged into the so-called 'average difference'. To normalize the data, the average differences were adjusted to produce an average intensity that equaled 2500.

To be certain of the estrogen-related changes in gene expression revealed by the DNA microarray analysis, we independently repeated the same 
experiment at least twice and the differences in intensities were calculated. To maintain reproducibility, we selected for further analysis only those genes whose expression levels at the same condition in two separate experiments did not change by more than twofold. The average values yielded by these independent experiments were then used to denote the gene expression levels. Calculation of Pearson's correlation coefficients and Fisher's z transformation were performed by StatView software (SAS Institute Inc., Cary, NG, USA).

Filtering of genes and clustering analysis were performed by using the GeneCluster program based on self-organizing maps (SOMs) developed by Tamayo et al. (1999).

\section{Quantitative-real time-PCR}

Total RNA was purified as described above. cDNA was synthesized from purified total RNA by Superscript II RT(-) (Invitrogen) with random primers at $42{ }^{\circ} \mathrm{C}$ for $60 \mathrm{~min}$. PCR reactions were performed in the PE Prism 5700 sequence detector (PE Biosystems, Tokyo, Japan) using the SYBRGreen PCR Core Reagents (PE Biosystems) in the presence of appropriate primers according to the manufacturer's instructions. Each PCR amplification was performed in triplicate using the following conditions: $2 \mathrm{~min}$ at $50{ }^{\circ} \mathrm{C}$ and $10 \mathrm{~min}$ at $95^{\circ} \mathrm{C}$, followed by 40 two-temperature cycles $(15 \mathrm{~s}$ at $95{ }^{\circ} \mathrm{C}$ and $1 \mathrm{~min}$ at $60{ }^{\circ} \mathrm{C}$ ). The use of a sequence detector allows continuous measurement of the fluorescent spectra emitted by SYBR-Green intercalated to double-stranded DNA during the PGR amplification. The model 5700 software constructed amplification plots from extension phase fluorescent emission data collected during PCR amplification. Threshold $(\mathrm{C} \tau)$ values were calculated by determining the point at which fluorescence exceeds a threshold limit (usually 10 times the S.D. of the baseline). The primers were chosen to amplify short PCR products (less than 100 base pairs) and their sequences are as follows: U51196 (APG-binding protein EBl homolog) CGCATTGCAACACAGAGGACTA, CGAGGA TTCTTTGGCACGAT; AJ005564 (small prolinerich (SPR) protein 2F) TGAGGGTTCAGCAACA ATGTCTT, TTGGTGGTGGACACACAGGA; D50086 (neuropilin) CAGCAAGCGCAAGGCTA AG, GAGGTGAAAACGTCGGAAGGT; L40406 (heat shock protein (hsp-E7I)) GGAACGACGGA
AAGTGTTGG, GCTCTGAAGTCGGCTGG AAT; X75285 (fibulin-2) CAGGTCGCATCAC GCACTAC, GGCGGATGGGGAAGATATG; AF087825 (claudin-7) CGCTGGTGTTGGGCT TCTTA, CAACAGGGTGTGCACTTGAT. To obtain standard curves of the genes, serial dilutions of a known amount of a cDNA sample were used. The $\mathrm{C} \tau$ values of each gene were plotted on these standard curves to obtain the amount of copies present in the initial cDNA sample and fold changes were calculated. Gene expression levels were normalized by the expression levels of $28 \mathrm{~S}$ (X00525) ribosomal RNA (primer sequences were AGACGGTGGTGAGAGAGGTTAGTT and GCAGGATTACGATGGCAACAA). Gel electrophoresis and melting curve analyses were performed to confirm correct amplicon size and the absence of nonspecific bands.

\section{Results}

\section{Reproducibility of the DNA microarray experiments}

In this study, we used DNA microarray analysis to examine the uterine gene expression in sesame oil-treated mice and in mice treated with four doses of physiological (E2) estrogen or two doses of non-physiological (DES) estrogen. In order to confirm the reproducibility of the DNA microarray data, the intensities of the genes expressed during the same conditions in two independent experiments were compared. As shown in Table 1, the gene expressions at all seven conditions in the two experiments were very similar, as the correlation coefficients were all greater than 0.8 and five of the seven were greater than $0 \cdot 9$. Thus, the microarray data were highly reproducible. In a previous paper, we used Student's $t$-test with three sets of independent DNA microarray experiments to identify 46 estrogen-induced genes (Watanabe et al. 2002). More than $80 \%$ of these genes were also found in this study (data not shown), which supports the reproducibility of these microarray experiments.

\section{Different effects of physiological and non-physiological estrogens on gene expression}

We first assessed the microarray data obtained with mice treated with $5 \mu \mathrm{g} / \mathrm{kg}$ b.w. of E2 or DES to 
Table 1 Correlation between the gene expression values obtained with two identically performed independent DNA microarray experiments. DNA microarray experiments with uterine RNA taken from oil-treated mice or mice treated with four doses of E2 or two doses of DES were repeated twice. The expression levels of the genes in both experiments were measured based on the Affymetrix software and the correlation coefficients between gene expression levels in the two experiments were calculated. The correlation coefficients are shown

\begin{tabular}{|c|c|c|c|c|}
\hline \multicolumn{5}{|c|}{ Dose of E2 or DES $(\mu \mathrm{g} / \mathrm{kg})$} \\
\hline 0 & 0.05 & 0.5 & 5 & 50 \\
\hline 0.93 & $\begin{array}{l}0.94 \\
\text { ND }\end{array}$ & $\begin{array}{l}0.94 \\
0.83\end{array}$ & $\begin{array}{l}0.87 \\
0.91\end{array}$ & $\begin{array}{l}0.93 \\
\text { ND }\end{array}$ \\
\hline
\end{tabular}

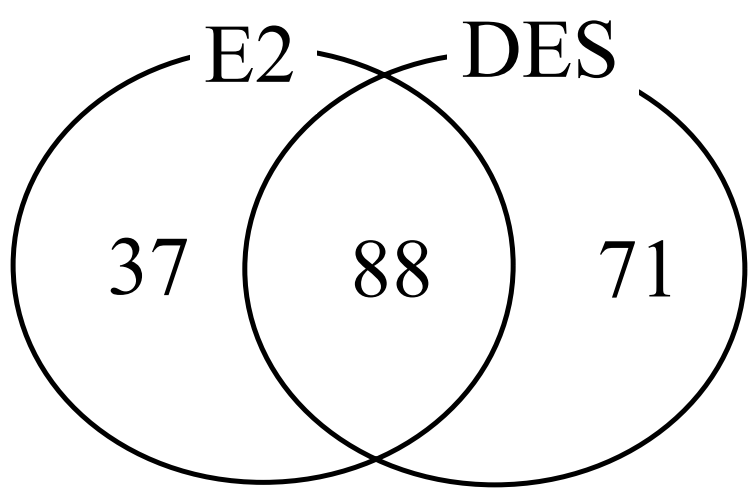

Figure 1 Venn diagram of the number of genes induced by E2 and DES. Ovariectomized mice were treated with $5 \mu \mathrm{g} / \mathrm{kg}$ of E2 or DES and total uterine RNAs were purified after $6 \mathrm{~h}$. Expression analysis was performed and the fold changes of gene expression levels were calculated based on the sesame oil control expression levels. The genes that were activated by either E2 or DES or both E2 and DES were counted.

determine whether these estrogens activate the same genes. The number of genes that were activated more than threefold by the estrogens was counted. We found that, in addition to the genes commonly activated by both estrogens (88 genes), some genes were activated by only one of the estrogens (37 genes for E2 and 71 for DES) (Fig. 1). This suggests that E2 and DES are not identical in their gene activation behavior despite a study with yeast reporter assays that revealed the two estrogens had a very similar effect on the genes examined (Korach et al. 1985, 1991, Coldham et al. 1997).

\section{Dose-dependent activation of genes by physiological and non-physiological estrogens}

The identification of genes activated by only one of the two estrogens prompted us to examine the dose-dependent uterine gene-expression patterns resulting from E2 or DES treatment. Thus, we examined the microarray data obtained from oil-treated mice and mice treated with four doses of E2 or two doses of DES. The expression levels recorded for each gene at each estrogen dose in the two experiments were averaged and analyzed by using the GeneCluster program based on self organizing maps (Tamayo et al. 1999). This analysis revealed that the expression of estrogen-activated genes as well as estrogen-repressed genes is dependent on the E2/DES dosage used (Fig. 2). Three additional features were also noted from the clustering analysis. First, the in vivo activity of DES in altering gene expression is much higher than that of E2. This correlates with the estrogenic activities of E2 and DES estimated by the prepubertal mouse (Coldham et al. 1997), although the extent of the difference was greater than the result in prepubertal mouse. The difference between the two systems may be due to the presence in vivo of binding proteins such as steroid hormone-binding globulin, which binds to DES more weakly than it binds to E2 (Sheehan \& Young 1979), causing more DES to be available for ER binding. Secondly, the genes differed in their dose-dependent activation/repression patterns as we observed that the estrogens induced at least two different dose-response patterns. Some gene clusters, such as classes C, D, G and H, were maximally activated or repressed at the highest estrogen doses used (for example, $50 \mu \mathrm{g} / \mathrm{kg}$ E2, Fig. 2) whereas the genes in classes A, E, and F were maximally activated or repressed at the middle of the dose range (for example, $5 \mu \mathrm{g} / \mathrm{kg}$ E2, Fig. 2). The latter pattern may reflect the well-known phenomenon of antagonism resulting from an overdose of the agonist. These observations suggest that the maximal activity of transcription differs among genes. These observations also suggest that the difference between E2 and DES in the number of the genes they activated by more than threefold at $5 \mu \mathrm{g} / \mathrm{kg}$ doses (Fig. 1) mainly reflects the difference in the relative activity of each estrogen and the difference in the dose-response patterns of the genes. Thirdly, the 

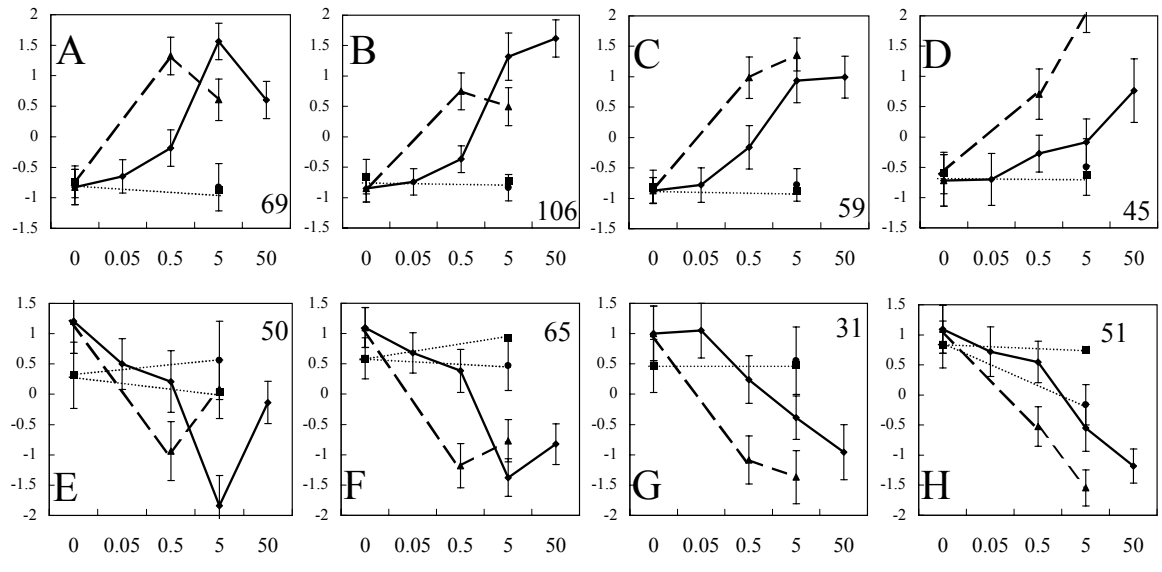

Figure 2 Clustering pattern of the genes whose expression is affected by E2 or DES. Based on the DNA microarray data, the dose-dependent gene expression patterns were clustered by a GeneCluster program based on self-organizing maps algorithm (see Materials and methods) and the clusters showing genes that are activated or repressed by both estrogens are indicated. Each graph $(\mathrm{A}-\mathrm{F})$ represents the dose-dependent $(\mu \mathrm{g} / \mathrm{kg}$; $X$ axis) expression pattern of a cluster represented by normalized values ( $\mathrm{Y}$ axis). The number of genes in each cluster is also indicated. Solid and dashed lines indicate the E2 and DES dose-responses as means of standardized gene expression levels respectively. The dotted line indicates the gene expression level of $\alpha$ ERKO mice. Standard deviations are also indicated.

extent of the gene activation induced by E2 or DES differs depending on the gene. Some genes had a higher maximal activity with DES than E2 whereas other genes showed the opposite pattern. For example, the genes in cluster B were induced by E2 to express much higher levels of message at the optimal E2 concentration compared with DES (Fig. 2). In contrast, in cluster D, DES induced a much higher maximal intensity of gene expression compared with E2 (Fig. 2). Some of the genes are shown with average intensities (Fig. 3). These gene-dependent differences were also confirmed by quantitative-real time-PGR (Q-RT-PGR) on selected genes (Fig. 3). For example, the APG-binding protein EB1 (U51196), the SPR protein $2 \mathrm{~F}$ (AJ005564) and neuropilin (D50086) were better activated by E2 than by DES, whereas HSP105 (L40406), fibulin-2 (X75285) and claudin-7 (AF087825) were more highly activated by DES compared with the same dose of E2. As the response was not at the maximal effective dose in all cases, the difference can be the result of the difference in the efficacy and potency of the estrogens. The biological significance of these
E2/DES-induced differences and the promoters involved should be examined further.

\section{Relationship between gene expression patterns and uterotropic activity}

As there were at least two types of dose-dependent gene activation patterns, such as those exemplified by clusters $\mathrm{A}$ and $\mathrm{B}$, we examined the relationship between these gene activation patterns and their uterotropic effect. Ovariectomized mice were treated with E2 for one week and the dosedependent uterotropic effects were estimated by weighing the uteri. We found that the greatest uterine weights occurred with the highest dose of E2 that was administered (Fig. 4A). This doseresponse does not match that of the genes in gene clusters such as A and E (Fig. 2), which are maximally up- or downregulated by the middle dose of E2. This suggests that these genes may not be directly involved in the uterotropic effect. In contrast, it is likely that the genes that have a dose-response similar to that of the uterotropic effect are directly involved in the uterotropism of 

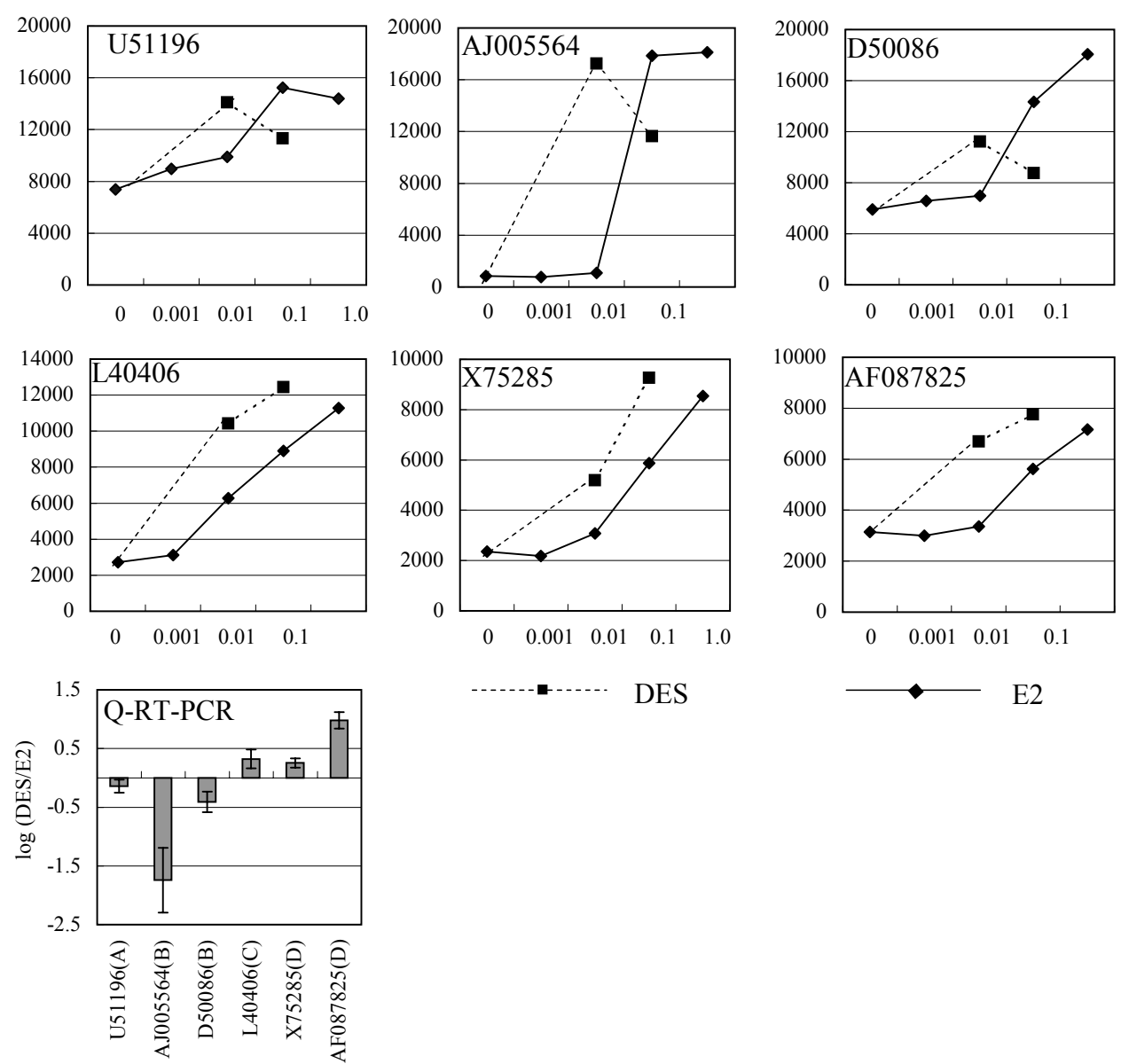

\section{DES}

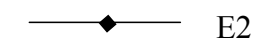

Figure 3 Quantitative PCR analysis of selected genes that are differently affected by DES and E2.The genes showing big differences in fold changes between E2 and DES were selected and dose curves of the selected genes were plotted from the microarray data. The $\mathrm{Y}$-axis indicates the average gene expression levels calculated from two independent experiments and the X-axis indicates the doses administered $(\mu \mathrm{g} / \mathrm{kg})$. The Genebank accession number is indicated in each panel. For Q-RT-PCR, ovariectomized mice were treated with $5 \mu \mathrm{g} / \mathrm{kg}$ of E2 or DES and uterine RNA was isolated after $6 \mathrm{~h}$. After cDNA synthesis, the fold changes in gene expression levels induced by the estrogens were estimated by quantitative-real time-PCR. After normalizing the expression relative to the expression levels of $28 \mathrm{~S}$ ribosomal RNA, the fold changes induced by DES were divided by the fold changes induced by E2. These ratios of fold changes are indicated by log2. Each experiment was repeated three times and the errors are indicated. The cluster classes to which the examined genes belong are indicated in parenthesis. U51196, APC-binding protein EB1 homolog; AJ005564, SPR protein 2F; D50086, neuropilin; L40406, Hsp105; X75285, fibulin-2; and AF087825, claudin-7.

E2. This notion is supported by the fact that these genes are not activated by $\mathrm{E} 2$ in $\alpha \mathrm{ERKO}$ mice (Fig. 2), nor does E2 have any uterotropic effects in these mice (Lubahn et al. 1993).

To select the genes that may be related to the uterotropic effect of E2, we calculated the correlation between the gene expression levels and the uterotropic effects associated with various doses of E2. We selected the genes associated with correlation coefficients that are over 0.85 (positively related genes) or under $-0 \cdot 85$ (negatively related genes) (Tables 2 and 3). We used these values for 

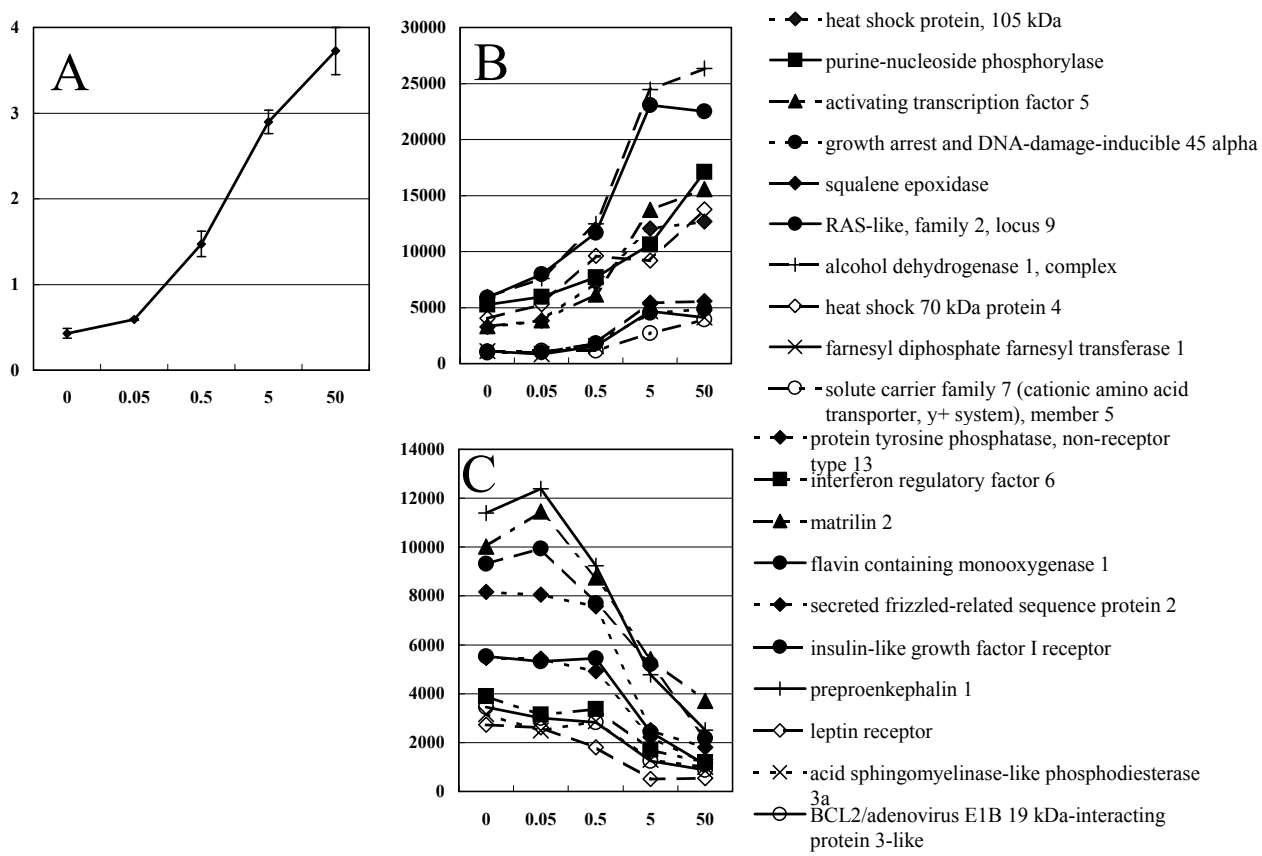

Figure 4 Correlation between gene expression levels and the uterotropic effect of varying E2 doses. For the uterotropic assay, ovariectomized mice were injected intraperitoneally with E2 or sesame oil every $24 \mathrm{~h}$ for 7 days and whole uteri $(n=5)$ were collected and weighed. (A) The ratio of uterine weight and body weight $(\mathrm{mg} / \mathrm{g})$ is indicated in Y-axis, X-axis indicates the doses administered $(\mathrm{mg} / \mathrm{kg})$. The correlation between the uterotropic effect and the expression of each gene at $6 \mathrm{~h}$ was calculated. Ten genes with high positive correlation coefficients $(>0.85)$ (B) and ten genes with high negative correlation coefficients (negatively-correlated genes) $(<-0.85)(C)$ were selected and their gene expression levels are indicated. The Y-axis indicates the gene expression levels estimated by DNA microarray and the $\mathrm{X}$-axis indicates the doses administered $(\mu \mathrm{g} / \mathrm{kg})$.

the selection because they gave positive values of $98 \%$ confidence intervals surrounding $\mathrm{r}$ after $\mathrm{z}$ transformation.

Included in the positively-correlated genes are stress-related proteins (Stip1, Hspa4, Hspa5 and Hsp105) and insulin-like growth factor I (Igf1). Igfl has previously been shown to be involved in the regulation of the estrogenic response (Murphy \& Ghahary 1990). Of the stress-related proteins, two are known chaperones and two others are related to chaperones. Stip $1 / \mathrm{mSTI} 1$, conserved from yeast to humans (Blatch et al. 1997), is known to interact with Hsp90 and Hsp70 (Lassle et al. 1997) and functions as a cochaperone. Hspa5/BiP is also known to have a chaperone-like activity (Hurtley et al. 1989, Flynn et al. 1991) at the endoplasmic reticulum (Dierks et al. 1993). The other stress proteins, Hspa4/apg-2 (Kaneko et al. 1997) and Hspl05 (Yasuda et al. 1995), are similar to hsp70RY but their functions are not yet known. Two transcription factors, Atf5 and Egr 1, were also activated. These transcription factors may be related to the activation of downstream genes. Also selected were two genes related to sterol synthesis, which we showed in an earlier study to be activated by estrogen (Watanabe et al. 2002). When we compared the positivly-correlated genes with temporal changes of the expression pattern (Watanabe et al. 2003), it was revealed that the expressions of the positively-correlated genes were not temporally correlated. For example, purinenucleoside phosphorylase was activated as an immediate-early gene, squalene epoxidase was activated as an early-late gene and heat shock protein was gradually activated (Watanabe et al. 2003).

With regard to the repressed genes, we found not only genes involved in metabolism but also genes 
Table 2 List of selected genes whose expression patterns are upregulated during the uterotropic effect of E2. The known genes whose E2-induced expression is highly positively correlated with the uterotropic effect of E2 are indicated. Correlation coefficients (CC), fold changes in E2-induced upregulation of gene expression relative to gene expression levels in oil-treated mice (FC), GenBank Accession numbers (GB\#), symbols and gene names are shown

\begin{tabular}{|c|c|c|c|c|}
\hline CC & FC & GB\# & Symbol & Gene Name \\
\hline 0.87 & $34 \cdot 31$ & X04480 & $\operatorname{lgf1}$ & insulin-like growth factor 1 \\
\hline 0.88 & $7 \cdot 01$ & AB013848 & Padi1 & peptidyl arginine deiminase, type I \\
\hline 0.90 & $5 \cdot 58$ & D85904 & Hspa4 & heat shock $70 \mathrm{kDa}$ protein 4 \\
\hline 0.86 & 5.48 & X75285 & Fbln2 & fibulin 2 \\
\hline 0.92 & 5.48 & D42048 & Sqle & squalene epoxidase \\
\hline 0.90 & $5 \cdot 21$ & AB017189 & Slc7a5 & solute carrier family 7 , member 5 \\
\hline 0.86 & $5 \cdot 15$ & U14648 & Sfrs 10 & splicing factor, arginine/serine-rich 10 \\
\hline 0.92 & 5.04 & U00937 & Gadd45 & growth arrest and DNA-damage-inducible 45 alpha \\
\hline 0.86 & 4.75 & M28845 & Egr1 & early growth response 1 \\
\hline 0.92 & 4.64 & AB012276 & Atf5 & activating transcription factor 5 \\
\hline 0.85 & 4.64 & L02526 & Map2k1 & mitogen activated protein kinase kinase 1 \\
\hline 0.91 & 4.42 & AJ002387 & Hspa5 & heat shock $70 \mathrm{kDa}$ protein 5 \\
\hline 0.92 & 3.91 & L32752 & Rasl2-9 & RAS-like, family 2 , locus 9 \\
\hline 0.93 & $3 \cdot 86$ & L40406 & Hsp105 & heat shock protein, $105 \mathrm{kDa}$ \\
\hline 0.86 & $3 \cdot 81$ & U59807 & Cstb & cystatin B \\
\hline 0.90 & $3 \cdot 62$ & D29016 & Fdft1 & farnesyl diphosphate farnesyl transferase 1 \\
\hline 0.90 & 3.41 & M22679 & Adh1 & alcohol dehydrogenase 1 , complex \\
\hline 0.85 & $3 \cdot 25$ & U27830 & Stip1 & stress-induced phosphoprotein 1 \\
\hline 0.92 & $3 \cdot 24$ & U35374 & Pnp & purine-nucleoside phosphorylase \\
\hline 0.85 & 3.05 & D89063 & Ddost & dolichyl-di-phosphooligosaccharide-protein glycotransferase \\
\hline
\end{tabular}

related to detoxification mechanisms such as cytochrome $\mathrm{P} 450$ and glutathione S-transferase. Moreover, we selected max dimerization protein 4 (Mad4) (Hurlin et al. 1995) and interferon regulatory factor 6 (Irf-6), which are known to be transcription factors. It may be that the downregulation of these transcription factors combined with the upregulation of the transcription factors noted above play an important role in mediating the responsiveness of the uterus to estrogen. Functional analysis of these selected genes should contribute to our understanding of the molecular mechanisms involved in the uterotropic effects of estrogens, particularly those evoked in the early stages.

\section{Discussion}

In this study, we showed that physiological and non-physiological estrogens do not exert identical effects on uterine gene expression. Although both estrogens generally induced similar genes, the expression of some genes was more profoundly affected by E2 than by DES (cluster B in Fig. 2) and vice versa (cluster D in Fig. 2). The fact that E2 and DES induce the expression of genes to different extents suggests that they may exert different physiological effects distinct from estrogen receptor binding activities.

Recently, it was found that the different estrogenic activities of estrogen and tamoxifen are due to their different requirements of a cofactor, steroid receptor coactivator 1 (SRG-1) (Shang \& Brown 2002), as the estrogen-like activity of tamoxifen requires high levels of SRC-1 expression whereas estrogen requires lower levels. This study also showed that while tamoxifen can recruit SRC-1 by binding to the ER, it is much less effective in recruiting other cofactors. Interestingly, the efficiency of this ligand-dependent recruitment of cofactors varies depending on the ligand and cofactors involved. For example, while ERs liganded with E2 and DES recruit SRC-3 equally efficiently, E2-liganded ERs can recruit SRC-1 and SRC-2 more efficiently than DES-liganded ERs (Bramlett et al. 2001). On the basis of these observations, we could propose that the different gene clusters revealed in this study may relate to the effect of these various coactivators. Although the difference between E2 and DES is not so 
Table 3 List of selected genes whose expression patterns are downregulated during the uterotropic effect of E2. The known genes whose E2-induced expression is highly negatively correlated with the uterotropic effect of E2 are indicated. Correlation coefficients (CC), fold changes in E2-induced upregulation of gene expression relative to gene expression levels in oil-treated mice (FC), GenBank Accession numbers (GB\#), symbols and gene names are shown

\begin{tabular}{|c|c|c|c|c|}
\hline CC & FC & GB\# & Symbol & Gene Name \\
\hline-0.91 & $-6 \cdot 75$ & U95826 & Ccng2 & cyclin G2 \\
\hline-0.86 & $-5 \cdot 80$ & J03953 & Gstm3 & glutathione S-transferase, mu 3 \\
\hline-0.88 & -5.59 & AB023957 & EIG180 & ethanol induced gene product EIG180 \\
\hline-0.93 & $-5 \cdot 34$ & U42467 & Lepr & leptin receptor \\
\hline-0.95 & -5.01 & D16215 & Fmol & flavin containing monooxygenase 1 \\
\hline-0.93 & -4.93 & M55181 & Penk1 & preproenkephalin 1 \\
\hline-0.97 & -4.71 & D83966 & Ptpn13 & protein tyrosine phosphatase, non-receptor type 13 \\
\hline-0.93 & -4.59 & AF056187 & $\operatorname{lgf} 1 \mathrm{r}$ & insulin-like growth factor I receptor \\
\hline-0.94 & -4.51 & U88567 & Sfrp2 & secreted frizzled-related sequence protein 2 \\
\hline-0.89 & -4.42 & U96724 & Pps & putative phosphatase \\
\hline-0.89 & -4.42 & AJ132192 & Hs1bp3 & HS1 binding protein 3 \\
\hline-0.88 & $-4 \cdot 38$ & U32395 & Mad4 & Max dimerization protein 4 \\
\hline-0.86 & $-4 \cdot 34$ & U09816 & Gm2a & GM2 ganglioside activator protein \\
\hline-0.85 & $-4 \cdot 27$ & M24417 & Abcb1a & ATP-binding cassette, sub-family $\mathrm{B}(\mathrm{MDR} / \mathrm{TAP})$, member $1 \mathrm{~A}$ \\
\hline-0.93 & -3.90 & AF067395 & Bnip31 & BCL2/adenovirus E1B 19 kDa-interacting protein 3-like \\
\hline-0.89 & -3.83 & X98055 & Gstt 1 & glutathione S-transferase, theta 1 \\
\hline-0.85 & -3.80 & U90435 & Flot1 & flotillin 1 \\
\hline-0.85 & -3.59 & M72394 & Pla2g4a & phospholipase A2, group IVA (cytosolic, calcium-dependent) \\
\hline-0.90 & -3.51 & L17022 & Fcgrt & Fc receptor, IgG, alpha chain transporter \\
\hline-0.92 & $-3 \cdot 36$ & X15052 & Ncam1 & neural cell adhesion molecule 1 \\
\hline-0.86 & -3.29 & AB033921 & Ndr2 & $\mathrm{N}$-myc downstream regulated 2 \\
\hline-0.91 & $-3 \cdot 28$ & D30779 & Pla2g1br & phospholipase A2, group IB, pancreas, receptor \\
\hline-0.97 & $-3 \cdot 26$ & U73029 & Irf6 & interferon regulatory factor 6 \\
\hline-0.87 & -3.23 & U07159 & Acadm & acetyl-coenzyme A dehydrogenase, medium chain \\
\hline-0.93 & $-3 \cdot 12$ & Y08135 & Asml3a & acid sphingomyelinase-like phosphodiesterase $3 a$ \\
\hline-0.87 & $-3 \cdot 11$ & L38444 & Tgtp & T-cell specific GTPase \\
\hline-0.95 & -3.08 & U69262 & Matn2 & matrilin 2 \\
\hline-0.92 & -3.08 & AJ007970 & Gbp2 & guanylate nucleotide binding protein 2 \\
\hline-0.87 & -3.05 & D50834 & Сур4b1 & cytochrome P450, subfamily IV B, polypeptide 1 \\
\hline
\end{tabular}

drastic as that between E2 and tamoxifen, it can be considered to have weak 'selective estrogen receptor modulator' activity.

Alternatively, the different estrogenic effects of various estrogens may also be due to non-genomic actions of the ER. It is known that signaling cascades such as extracellular signal-regulated kinase (ERK)/mitogen-activated kinase (MAPK) or the modulation of G-protein-coupled receptors are involved in the so-called non-genomic action of estrogen (see references in Levin 2003). Testing these possibilities to determine what is responsible for the different estrogenic effects of various estrogens may facilitate our understanding of the mechanism of estrogenicity.

In our previous study, we examined temporal changes of gene expression after estrogen administration (Watanabe et al. 2003). Although we compared the clustering result of the temporal changes and that of the estrogen response, we could not find a clear correlation between them. This result indicates that sensitivity and efficacy of estrogen are not directly related to temporal changes of the gene expression pattern.

In this study, it was shown that a group of genes showed a dose-response similar to that of the uterotropic effect. It suggests that we can select the genes that may be directly related to the uterotropic effect among a large number of estrogen-induced genes (Watanabe et al. 2002). Gene expression analysis of the selected genes can be helpful for understanding the inconsistency of estrogenic chemicals in ER binding activity and uterotropic activity. That the uterotropic effects of estrogenic chemicals can differ despite equivalent ER-binding activities in the human has long been 
known. For example, this phenomenon has been observed in the metabolites of DES, as indenestrol $\mathrm{A}$ and $\mathrm{B}$, indanyl-DES and Z-pseudo-DES all have higher ER binding activities but lower uterotropic effects compared with DES. DES and some of these chemicals show similar temporal nuclear distribution (Korach et al. 1985), which suggests that the different uterotropic effects of these agents may be due to their different gene activation patterns. Transcriptome analyses of the effect of the different DES metabolites will be helpful in testing this notion.

We used quantitative PCR to check the expression of several genes that were revealed by the DNA microarray experiments to be differently expressed in E2-treated uteri compared with DES-treated uteri. However, upon quantitative PCR analysis, some of these genes did not show the same patterns revealed by microarray analysis. We have also observed in our previous study that the fold changes in expression that are estimated by quantitative PCR do not always correlate with those estimated by DNA microarray experiments. These limitations in DNA microarray analysis not only block the accurate characterization of gene expression patterns, they also hinder clustering analysis. Thus, it is necessary to confirm the activation pattern detected by microarray analysis by quantitative PCR. Despite this limitation in DNA microarray assays, this technology still has major advantages in the evaluation of estrogenic activity relative to other methods. Although the yeast system is a conventional means used to assess the effect of estrogen on gene expression, it cannot evaluate the effect of estrogen-binding proteins that exist in vivo and it is difficult to evaluate the effect of estrogen on target tissues. Furthermore, while the uterotropic assay is a useful method for the evaluation of estrogenic activity in vivo, it is a time-consuming procedure. Thus, microarray analysis is an ideal method to evaluate the effect of estrogenic activity on gene expression patterns, as it is a quick method that assesses the estrogenic activity in vivo.

\section{Acknowledgements}

We thank Dr Caren Helbing for critically reading the manuscript and Eri Takahashi for technical assistance. This work was supported, in part, by a research grant from the Core Research for Evolutional Science of Japan Science and Technology Cooperation, a Grant-in-Aid from the Scientific Research of Japan Society for the Promotion of Science, a Grant-in-Aid for Scientific Research from the Ministry of Education, Science, Sports and Culture of Japan, a Health Sciences Research Grant from the Ministry of Health, Labor and Welfare of Japan, a Research Grant from the Ministry of Environment of Japan, and a Research Grant from the New Energy and Industrial Technology Development Organization (NEDO).

\section{References}

Blatch GL, Lassle M, Zetter BR \& Kundra V 1997 Isolation of a mouse cDNA encoding mSTI1, a stress-inducible protein containing the TPR motif. Gene 194 277-282.

Bramlett KS, Wu Y \& Burris TP 2001 Ligands specify coactivator nuclear receptor (NR) box affinity for estrogen receptor subtypes. Molecular Endocrinology 15 909-922.

Coldham NG, Dave M, Sivapathasundaram S, McDonnell DP, Connor C \& Sauer MJ 1997 Evaluation of a recombinant yeast cell estrogen screening assay. Environmental Health Perspectives 105 734-742.

Dierks T, Klappa P, Wiech H \& Zimmermann R 1993 The role of molecular chaperones in protein transport into the endoplasmic reticulum. Philosophical Transactions of the Royal Society of London Series B. Biological Sciences 339 335-341.

Dodds EC, Goldberg L, Lawson W \& Robinson R 1938 Oestrogenic activity of certain synthetic compounds. Nature 141 247-249.

Feng X, Jiang Y, Meltzer P \& Yen PM 2000 Thyroid hormone regulation of hepatic genes in vivo detected by complementary DNA microarray. Molecular Endocrinology 14 947-955.

Flores-Morales A, Gullberg H, Fernandez L, Stahlberg N, Lee NH, Vennstrom B \& Norstedt G 2002 Patterns of liver gene expression governed by TRbeta. Molecular Endocrinology 16 1257-1268.

Flynn GC, Pohl J, Flocco MT \& Rothman JE 1991 Peptide-binding specificity of the molecular chaperone BiP. Nature 353 726-730.

Hurlin PJ, Queva C, Koskinen PJ, Steingrimsson E, Ayer DE, Copeland NG, Jenkins NA \& Eisenman RN 1995 Mad3 and Mad4: novel Max-interacting transcriptional repressors that suppress c-myc dependent transformation and are expressed during neural and epidermal differentiation. EMBO fournal 14 $5646-5659$

Hurtley SM, Bole DG, Hoover-Litty H, Helenius A \& Copeland CS 1989 Interactions of misfolded influenza virus hemagglutinin with binding protein (BiP). Fournal of Cell Biology 108 2117-2126.

Kaneko Y, Kimura T, Kishishita M, Noda Y \& Fujita J 1997 Cloning of apg-2 encoding a novel member of heat shock protein 110 family. Gene 189 19-24.

Kimura T, Basu SL \& Nandi S 1967 Nature of induced persistent vaginal cornification in mice. I. Effect of neonatal treatment with various doses of steroids. Fournal of Experimental Zoology 165 71-87.

Korach KS, Fox-Davies C, Quarmby VE \& Swaisgood MH 1985 Diethylstilbestrol metabolites and analogs. Biochemical probes for differential stimulation of uterine estrogen responses. Fournal of Biological Chemistry 260 15420-15426.

Korach KS, Chae K, Gibson M \& Curtis S 1991 Estrogen receptor stereochemistry: ligand binding and hormonal responsiveness. Steroids 56 263-270. 
Lassle M, Blatch GL, Kundra V, Takatori T \& Zetter BR 1997 Stress-inducible, murine protein mSTI1. Characterization of binding domains for heat shock proteins and in vitro phosphorylation by different kinases. Fournal of Biological Chemistry 272 1876-1884.

Levin ER 2003 Bidirectional signaling between the estrogen receptor and the epidermal growth factor receptor. Molecular Endocrinology 17 309-317.

Lockhart DJ, Dong H, Byrne MC, Follettie MT, Gallo MV, Chee MS, Mittmann M, Wang C, Kobayashi M, Horton H \& Brown EL 1996 Expression monitoring by hybridization to high-density oligonucleotide arrays. Nature Biotechnology 14 1675-1680.

Lubahn DB, Moyer JS, Golding TS, Couse JF, Korach KS \& Smithies O 1993 Alteration of reproductive function but not prenatal sexual development after insertional disruption of the mouse estrogen receptor gene. PNAS 90 11162-11166.

Murphy LJ \& Ghahary A 1990 Uterine insulin-like growth factor-I: regulation of expression and its role in estrogen-induced uterine proliferation. Endocrine Reviewes 11 443-453.

Shang Y \& Brown M 2002 Molecular determinants for the tissue specificity of SERMs. Science 295 2465-2468.
Sheehan DM \& Young M 1979 Diethylstilbestrol and estradiol binding to serum albumin and pregnancy plasma of rat and human. Endocrinology 104 1442-1446.

Tamayo P, Slonim D, Mesirov J, Zhu Q, Kitareewan S, Dmitrovsky E, Lander ES \& Golub TR 1999 Interpreting patterns of gene expression with self-organizing maps: methods and application to hematopoietic differentiation. PNAS 96 2907-2912.

Watanabe H, Suzuki A, Mizutani T, Khono S, Lubahn DB, Handa H \& Iguchi T 2002 Genome-wide analysis of changes in early gene expression induced by oestrogen. Genes Cells 7 497-507.

Watanabe H, Suzuki A, Kobayashi M, Takahashi E, Itamoto M, Lubahn DB, Handa H \& Iguchi T 2003 Analysis of temporal changes in the expression of estrogen-regulated genes in the uterus. Fournal of Molecular Endocrinology 30 347-358.

Yasuda K, Nakai A, Hatayama T \& Nagata K 1995 Cloning and expression of murine high molecular mass heat shock proteins, HSP105. Fournal of Biological Chemistry 270 29718-29723.

Received in final form 15 May 2003 Accepted 2 September 2003 